\title{
Neutron diffractive imaging of the skyrmion lattice nucleation in $\mathrm{MnSi}$
}

\author{
T. Reimann, ${ }^{1,2,{ }^{*}}$ A. Bauer, ${ }^{3}$ C. Pfleiderer, ${ }^{3}$ P. Böni, ${ }^{2}$ P. Trtik, ${ }^{4}$ A. Tremsin, ${ }^{5}$ M. Schulz,${ }^{1,2}$ and S. Mühlbauer ${ }^{1, \dagger}$ \\ ${ }^{1}$ Heinz, Maier-Leibnitz Zentrum (MLZ), Technische Universität München, Lichtenbergstrasse 1, Garching, Germany \\ ${ }^{2}$ Physik-Department E21, Technische Universität München, James-Franck-Strasse 1, Garching, Germany \\ ${ }^{3}$ Physik-Department E51, Technische Universität München, James-Franck-Strasse 1, Garching, Germany \\ ${ }^{4}$ Neutron Imaging and Activation Group, Laboratory for Neutron Scattering and Imaging, Paul Scherrer Institut, Villigen PSI, Switzerland \\ ${ }^{5}$ Space Sciences Laboratory, University of California, Berkeley, California, USA
}

(Received 21 August 2017; revised manuscript received 17 December 2017; published 12 January 2018)

\begin{abstract}
Spatially resolved neutron diffractive imaging with a microchannel plate collimator is used to directly map the nucleation of the skyrmion lattice (SkL) of the B20 compound MnSi at the conical to SkL transition as a function of magnetic field. Our study shows a macroscopic phase separation of the SkL and the conical phase at the border of the SkL phase pocket, reveals that the nucleation of the SkL starts at the edges of the sample, and quantifies the bending of the SkL due to demagnetization. Our study highlights the importance of geometric and demagnetizing effects for the SkL formation regarding the unambiguous interpretation of measurements of bulk properties such as possible phase coexistence and crossover regimes.
\end{abstract}

DOI: 10.1103/PhysRevB.97.020406

Recently, magnetic skyrmions in chiral magnets have attracted vast interest. Together with the ease of manipulating skyrmions at ultrasmall current densities [1], their topology ideally suits future devices for data storage and spintronics [2-5]. First identified in the B20 compounds MnSi and $\mathrm{FeCo}_{1-x} \mathrm{Si}_{x}$ [6,7], similar textures have been observed in various materials [8,9], including metals [8], semiconductors [7], and insulators $[10,11]$. The findings of skyrmions beyond the B20 family [12-15] and their observation in thinned bulk samples [16-18], thin films [19], and nanostructured patterns [20] show that magnetic skyrmions are a generic phenomenon of materials which promote chiral magnetic interactions. In the $A$-phase of $\mathrm{MnSi}$, skyrmions emerge as a densely packed hexagonal skyrmion lattice (SkL) which is distinct from the adjacent conical phase (CP) because of its finite topological winding number [6]. The topological difference of the SkL phase and the $\mathrm{CP}$ supports long-living metastable and supercooled skyrmion phases [21,22] and raises the generic question of the nucleation and decay of skyrmions at the borders of the SkL phase pocket [21], where a first-order phase transition is observed [23]. Magnetic force microscopy (MFM) suggests that skyrmion lines decay by means of point defects which act similarly to the slider of a zipper [21]. Recent studies using Lorentz transmission electron microscopy (LTEM) find an exponential decay of skyrmions suggesting enthalpy-entropy compensation [24]. Moreover, terms induced by free surfaces [25,26] and interface spin orbit terms $[3,19]$ seem to play a pivotal role.

Mapping out the phase diagram for $T<T_{c}$, the skyrmion phase is found to be enclosed by a region where susceptibility, specific heat [27], magnetotransport [28], and small-angle neutron scattering (SANS) [29] suggest phase coexistence of SkL and CP. The peculiarities of this transition region have

\footnotetext{
*tommy.reimann@frm2.tum.de

†sebastian.muehlbauer@frm2.tum.de
}

been debated intensively [30,31], e.g., in terms of a phase of Abrikosov-like magnetic vortices [28] or as consisting of individual skyrmion clusters [29]. However, in all cases, a clear correlation between bulk measurements and the intrinsic microscopic behavior of the $\mathrm{SkL}$ is hindered due to geometric effects caused by the nonellipsoidal sample shape: In this context, it has been shown that the extent of the transition region is influenced by the sample geometry [27]. In particular, edge instabilities delaying a skyrmion formation have been reported [32]. In contrast, for direct space mapping techniques using microscopy [16-18,21], the limited field of view, the limitation to thin(ned) samples, and the associated increasing influence of surface terms do not allow the unambiguous link with intrinsic microscopic behavior. Effects on a macroscopic scale like phase separation remain invisible.

In this paper, we systematically study the influence of sample geometry on the SkL nucleation, distribution, and distortion in bulk $\mathrm{MnSi}$ samples using spatially resolved neutron diffractive imaging (nDI) combined with susceptibility measurements. While this method is well established to map the bulk distribution and crystallite orientation of single and polycrystalline materials [33], its application to long-range magnetic spin structures such as the SkL was previously not possible due to the small Bragg angles involved. This obstacle has been overcome thanks to the implementation of a microchannel plate (MCP) acting as a collimator [34] to the imaging setup. Our approach allows us to directly map the nucleation and distribution of a SkL for bulk samples with sub$\mathrm{mm}$ position resolution. We find clear hints for a macroscopic phase separation arising at the border of the SkL-phase with SkL nucleation at the sample edges. A strong increase of the mosaic of the SkL toward the edge of the samples is found, mainly caused by demagnetizing effects. Both observations naturally account for the magnetic properties under debate, observed in bulk measurements at the border of the SkL phase. Our data underline that any conclusion on the SkL nucleation 
(a)

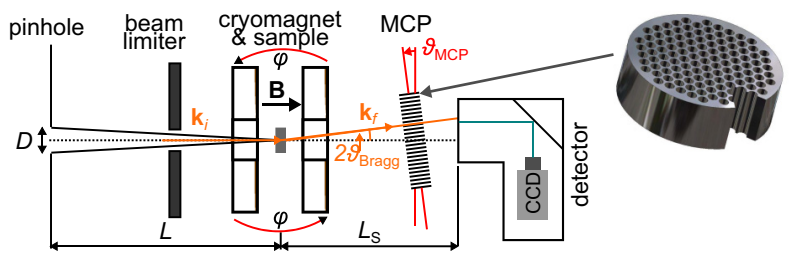

(b)

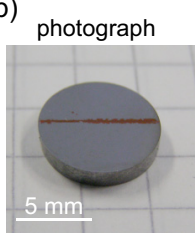

(c)

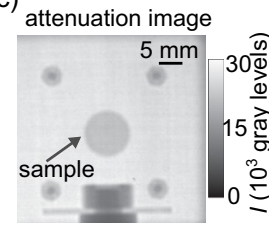

(d)

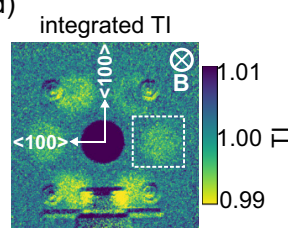

FIG. 1. (a) Illustration of the nDI setup (not to scale). The direct beam is blocked by an MCP; only neutrons Bragg scattered from the SkL reach the detector. $\varphi$ designates the rocking angle. (b) Photograph of the MnSi disk (sample 1). (c) Neutron absorption image (without MCP) of sample 1 in its holder measured at $T>T_{C}$. (d) Sum of 20 neutron images as measured in the SkL-phase for 20 rocking angles $-2.5^{\circ} \leqslant \varphi \leqslant 1.3^{\circ}$. For these measurements, the MCP was removed. Because of the normalization with the scattering in the paramagnetic phase, the signal is purely magnetic. The Bragg spot enclosed in the white square was investigated by $\mathrm{nDI}$.

and decay requires an extremely careful consideration of geometric effects for bulk samples.

The nucleation of the SkL was studied in MnSi using a disk sample [sample 1: thickness $t=2.1 \mathrm{~mm}$, diameter $d=9.3 \mathrm{~mm}$, Fig. 1(b)] with a crystallographic [100] direction normal to the disk. A small cuboid (sample $2: 1 \times 1 \times 1 \mathrm{~mm}^{3}$ ) and a small flat cube (sample 3:5 $\times 2 \times 1 \mathrm{~mm}^{3}$, [100] parallel to the shortest edge) of the same crystal was used to determine the magnetic phase diagram by means of magnetization measurements using a physical properties measurement system (PPMS, Quantum Design). The samples were wire cut from single crystals that had been grown by the floating zone method [27] with a typical residual resistivity ratio (RRR) of 80 [35]. The flat faces have been polished to remove damages introduced by the cutting process.

The nDI setup was implemented at the ANTARES beamline at the Heinz Maier-Leibnitz Zentrum (MLZ) [36]. The sample was located at a distance $L=8.3 \mathrm{~m}$ from the pinhole, and the distance between the sample and ${ }^{6} \mathrm{LiF}$ scintillator screen was $L_{\mathrm{S}}=54 \mathrm{~cm}$. The scintillator light from the absorption of the neutrons was recorded using a charge-coupled device (CCD) camera. The pinhole diameter $D=18 \mathrm{~mm}$ yields an $L / D$ ratio of 460 . The neutron wavelength $\lambda=4.0 \AA$ was selected by means of a neutron velocity selector (bandwidth $\Delta \lambda / \lambda=0.1)$. The sample was mounted at the cold finger of a closed-cycle cryostat attached to a normal conducting magnet with B parallel to the surface normal [100] of the sample and parallel to the wave vector $\mathbf{k}_{i}$ of the incident beam. The entire setup could be rotated by $\varphi$ [Fig. 1(a)].

To entirely discriminate neutrons that are Bragg scattered at the SkL from the direct beam, the MCP (placed in front of the detector) is rotated with respect to $\mathbf{k}_{i}$ by the scattering angle of a selected Bragg peak of the SkL, i.e., $\vartheta_{\mathrm{MCP}}=$ $2 \vartheta_{\text {Bragg }} \approx 1.2^{\circ}$. The MCP consists of glass fibers enriched with
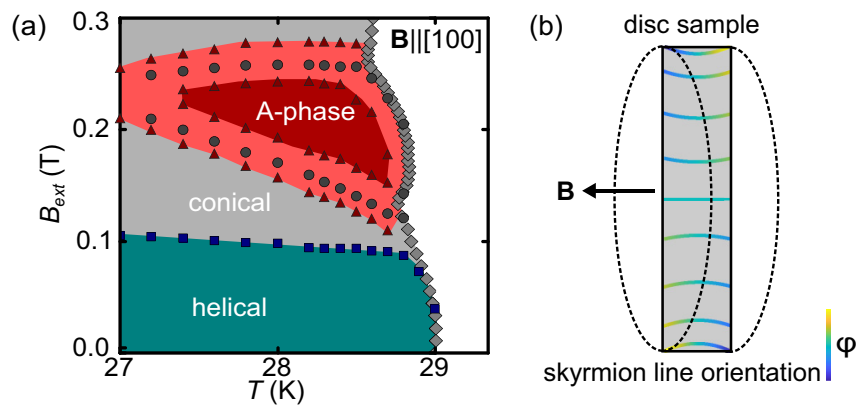

FIG. 2. (a) Magnetic phase diagram of MnSi in the vicinity of the SkL-phase (sample 2, B $\|$ [100]). (b) Schematic visualization of the bending of the SkL inside the sample, caused by geometry and demagnetization. Near the edge, the SkL fulfills the Bragg condition over a broader angular range of $\varphi$.

a mixture of $\mathrm{Gd}_{2} \mathrm{O}_{3}$ and $\mathrm{B}_{2} \mathrm{O}_{3}$ that are hexagonally packed with a lattice constant $a_{\text {hex }}=10 \mu \mathrm{m}$. Within the fibers, pores of $8 \mu \mathrm{m}$ diameter had been etched, forming the microchannels. Because of the composition of the glass, the MCP reveals a high absorption for cold neutrons outside the pores. It thus acts as a thin, well-defined neutron collimator with an angular divergence of $0.23^{\circ}$ [37].

Before investigating the SkL using $\mathrm{nDI}$, we have characterized the $(B, T)$-phase diagram of samples 2 and 3 for $\mathbf{B} \|[100]$ [Fig. 2(a)]. The phase boundaries were obtained from the derivative of the magnetization $\frac{d M}{d B}$ following Ref. [27]. The SkL phase (dark red) is characterized by a plateau of $\frac{d M}{d B}$ while the transition into the SkL phase is characterized by a broad peak in $\frac{d M}{d B}$. The onsets of the peak and the peak positions are represented by the red triangles and as gray circles, respectively, and form the transition region shaded in light red. In order to account for their different sample shapes, magnetic fields applied to samples 2 and 3 were rescaled by their demagnetizing factors $D_{2}$ and $D_{3}$ to the external field scale of the disk sample (sample 1, demagnetizing factor $D_{1}$ ) following Eq. (1.6) of Ref. [9] with the magnetic susceptibility $\chi_{\text {con }}$ within the CP.

A neutron radiography of sample 1 in its holder is shown in Fig. 1(c). Above $T_{\mathrm{C}}$, the contrast is solely generated by the variation in neutron absorption. When the sample enters the SkL phase, small additional contrast arises due to Bragg scattering of neutrons by the SkL. By rotating the sample-field assembly with respect to the vertical axis (rocking scan), the Bragg condition will be fulfilled for $\varphi=\vartheta_{\text {Bragg. }}$. Figure 1(d) shows the sum of 20 transmission images (TI) of the $\mathrm{MnSi}$ disk measured at equiangular positions for $-2.5^{\circ} \leqslant \varphi \leqslant 1.3^{\circ}$ at $T=28 \mathrm{~K}$ and $B=200 \mathrm{mT}$. The MCP was removed for this image, and hence all six Bragg spots are visible, corresponding to the hexagonally aligned Bragg peaks observed in SANS [6].

In contrast to SANS where $L \simeq 20 \mathrm{~m}$, the small sample-todetector distance $\left(L_{S}=54 \mathrm{~cm}\right)$ allows us to directly correlate each pixel of the diffraction spots to a specific position of the sample perpendicular to the beam axis. The inserted MCP collimator, which is carefully adjusted parallel to the wave vector $\mathbf{k}_{f}$ of the diffracted neutrons [Fig. 1(a)] for one of the six Bragg peaks of the $\mathrm{SkL}$ removes the direct beam and improves 
(a)

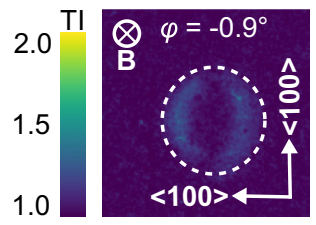

(b)
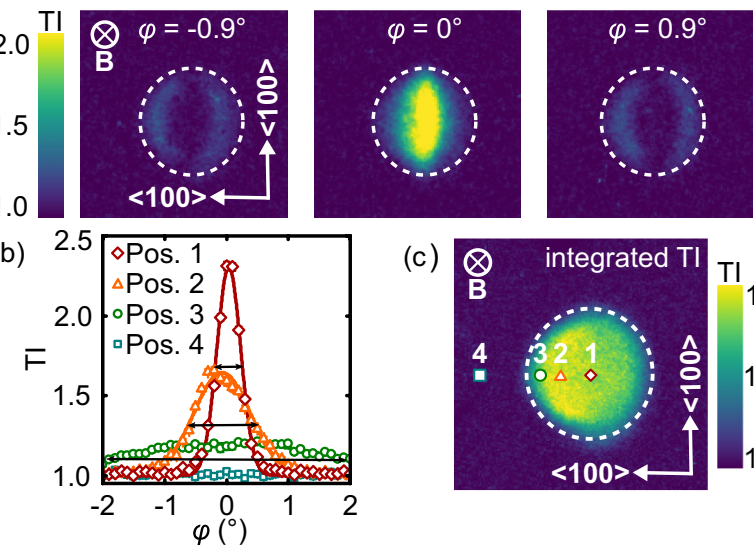

(c)

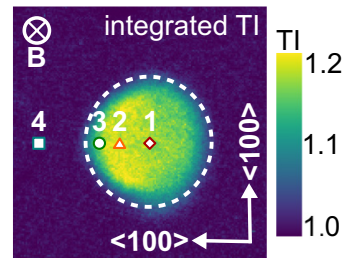

FIG. 3. $\varphi$ dependence of the diffraction spot enclosed by the white square in Fig. 1(d) ( $T=28 \mathrm{~K}, B=200 \mathrm{mT}$, exposure time $300 \mathrm{~s})$. The white circles in panels (a) and (c) indicate the shape of the sample. (a) Diffraction images shown for $\varphi=-0.9^{\circ}, 0^{\circ}$, and $0.9^{\circ}$. (b) Angular dependence of the intensity at the positions 1-4 as defined in panel (c). The rocking width increases strongly from the center of the sample (pos. 1) to its edge (pos. 3). Error bars are smaller than the symbols. The slight shift of the center of the rocking curves of $\approx 0.2^{\circ}$ is caused by the finite homogeneity of the magnetic field. (c) Sum of 41 single diffraction images obtained for $-2.0^{\circ} \leqslant \varphi \leqslant 2.0^{\circ}$.

the signal-to-noise ratio to enable a quantitative investigation of the spatial distribution of the SkL phase in the sample.

Results of $\mathrm{nDI}$ from the SkL of the MnSi disk are shown in Figs. 3(a)-3(c) for the Bragg peak enclosed by the white square in Fig. 1(d). A magnetic field $B=200 \mathrm{mT}$ was applied after cooling the sample in zero field to $T=28 \mathrm{~K}$, which brings the sample to the center of the $A$-phase. The color-coded diffraction image for each $\varphi$ shows at which position of the sample the Bragg condition for the SkL is fulfilled. Note that the center position of the rocking scans is set to $\varphi_{\text {Bragg }} \equiv 0^{\circ}$ to simplify the notation. The parasitic background arising from the scintillator structure and the sample holder was removed by subtracting the data measured at $B=260 \mathrm{mT}$, where no signature of the $\mathrm{SkL}$ is detected anymore. Fits to the data show that the SkL is aligned along $\mathbf{B}\left(\varphi=0^{\circ}\right)$ in the center of the sample while the SkL is not parallel to $B$ anymore and strongly bent near the left and right boundary of the sample $\left(\varphi= \pm 0.9^{\circ}\right)$ as indicated by the sickle-shaped pattern [38].

Figure 3(b) shows the intensity as obtained by evaluating the data at four different positions on the sample as marked in Fig. 3(c) as function of $\varphi$. The data correspond to SANS rocking scans, however, providing the spatially resolved angular distribution of the SkL. The curves can be well approximated by a Gaussian peak (solid lines). A strong broadening of the rocking curves from the center of the sample (pos. 1) towards the edge (pos. 3) is observed, accompanied by an increase of the FWHM from $0.4^{\circ}$ (1), representing the resolution limit, to $3.8^{\circ}$ (3) at the boundary. Position (4) provides the background level. The observed broadening of the rocking curves, which manifests in the sickle-shaped diffracted images, can be explained by a strong bending of the SkL at the sample edges due to inhomogeneous demagnetization fields as schematically illustrated in Fig. 2(b), taking the weak net FM moment into account. The data indicate radii of curvature of
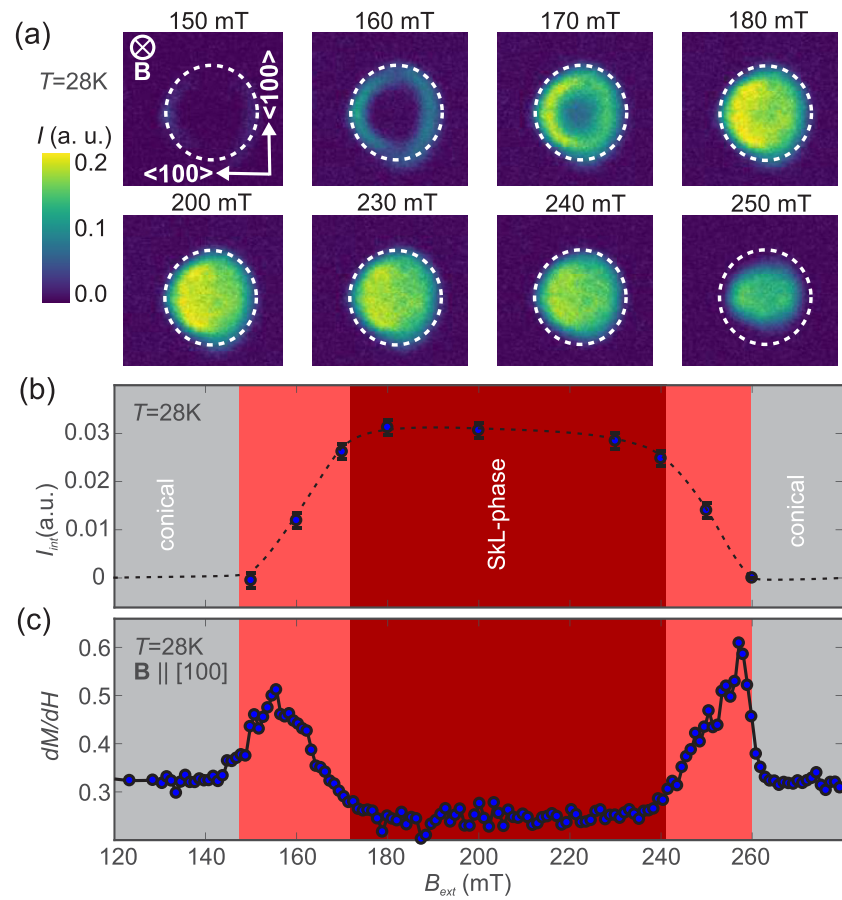

FIG. 4. (a) Field-dependent distribution of the skyrmion phase when crossing the boundaries of the SkL phase at $T=28 \mathrm{~K}$. Shown are integrated transmission images obtained similarly as in Fig. 3(c) for $150 \mathrm{mT} \leqslant B \leqslant 250 \mathrm{mT}$. It is only at intermediate fields where the sample is fully in the skyrmion phase. (b) Scattering intensity integrated over the entire sample $I_{\mathrm{int}}$ as function of $B$. The line is a guide to the eye. (c) $d M / d H$ taken on sample 3 at $T=28 \mathrm{~K}$, scaled by $D$.

the SkL of a few centimeters at the samples edges. Figure 3(c) shows the integrated intensity of 41 single diffraction images obtained for equidistant steps $-2.0^{\circ} \leqslant \varphi \leqslant 2.0^{\circ}$.

Finally, the nucleation of the SkL for increasing magnetic field was investigated using nDI. Figure 4(a) shows diffraction images as obtained by integrating 41 rocking angles $-2^{\circ} \leqslant$ $\varphi \leqslant 2^{\circ}$ using an exposure time of 300s for each setting for fields $150 \mathrm{mT} \leqslant B \leqslant 250 \mathrm{mT}$ applied after zero field cooling (ZFC) to $28 \mathrm{~K}$. The data have been normalized to $B=260 \mathrm{mT}$ at which no signature of the SkL was detected anymore. The intensity integrated over the entire sample volume $I_{\text {int }}$ is depicted in Fig. 4(b).

At the lower boundary of the $\mathrm{SkL}$ phase, i.e., $B=150 \mathrm{mT}$, weak intensity arises at the left and right edges of the disk, indicating the onset of SkL nucleation. With increasing $B$, a ring of intensity develops at the edge of the sample accompanied by a steep increase of $I_{\text {int }}$. Finally, between $180 \mathrm{mT}$ and $230 \mathrm{mT}$, Bragg scattering from the SkL is detected all over the sample, indicating that the SkL homogeneously fills the whole sample. Similarly, $I_{\text {int }}(B)$ levels off at $200 \mathrm{mT}$, confirming the stabilization of the SkL-phase throughout the whole sample. Finally, near the upper boundary of the SkL-phase pocket, the SkL begins to shrink toward the center of the sample, accompanied by a steep decrease of $I_{\text {int }}$ above $240 \mathrm{mT}$. While at $250 \mathrm{mT}$ scattering off the SkL arises merely from the center, no signature of the SkL has been detected anymore at $260 \mathrm{mT}$, proving the completed transition back to the $\mathrm{CP}$. The data 
at $260 \mathrm{mT}$ have been used for background subtraction as no signature of the SkL has been measured. As can be seen from the subtraction, the integral intensity is identical to data at $150 \mathrm{mT}$ where $I_{\text {int }}$ is found to lie below the systematical and statistical detection limit. Figure 4(c) shows $d M / d H$ taken on sample 3 at $T=28 \mathrm{~K}$. All data are scaled to the external field scale by the demagnetization factor.

The nDI data directly visualize the inhomogeneous nucleation of a SkL in a macroscopic, nonellipsoidal bulk sample. Two major effects may be identified: (i) the SkL first nucleates at the edge of the sample due to the field enhancement caused by demagnetizing fields. The SkL follows the internal magnetic field which results from a superposition of the applied and demagnetizing field, leading to a bending of skyrmion lines. An internal field profile as sketched in Fig. 2(b) seems likely considering the overall net FM moment of the CP and SkL [39]. (ii) Demagnetization provokes an extended region of macroscopic phase coexistence of CP and SkL at the borders of the $A$ phase. The region where macroscopic phase separation is observed by $\mathrm{nDI}[$ Fig. 4(a)] exactly tracks position and width of the transition region (broad peaks) observed by bulk $d M / d H$ measurements [Fig. 4(c)] as well as the slopes of $I_{\text {int }}(B)$ [Fig. 4(b)] at both the lower and the upper field boundaries of the SkL phase. The field dependence of $I_{\text {int }}(B)$ coincides with the integrated intensity routinely observed by SANS $[29,40]$. As rescaling according to demagnetizing factor affects only the position and not the relative width of a phase transition, our data consistently show that the extended crossover region is in fact caused by an incomplete volume filling of the sample with SkL (samples 1 and 3 have a similar demagnetization factor). Note that neutron diffraction in general only tracks the formation of a SkL phase which shows at least a certain amount of short-range order. A completely disordered SkL state would be invisible to our setup. Moreover, due to the scattering geometry, also the $\mathrm{CP}$ is not visible.

Our measurements clearly demonstrate the strong impact of sample geometry on bulk measurements such as magnetization and susceptibility but also neutron scattering, which are usually obtained as average over the whole sample. Our spatially resolved data show that even far from the borders of the sample, demagnetization effects may lead to a considerable smearing or shift of a thermodynamic phase transition. Accordingly, also magnetoresistivity and Hall-effect measurements might be affected by a coexistence of $\mathrm{CP}$ and SkL phase. It is very likely that the long-debated transition region [28-30] is in fact the hallmark of a macroscopic phase separation. In particular, the phase coexistence of SkL and CP may be misinterpreted as rounding of the first-order phase transition in bulk measurements or as precursor or mesophase effects [31,41].

Recent progress in theory and LTEM measurements of SkL formation showed attractive skyrmion interaction and clustering, in particular related to the presence of surfaces in thinned samples [26]. However, our study shows that similarly to superconducting vortices [42], theoretical modeling of the SkL must implicitly include preferred nucleation centers such as corners and edges in order to provide a sufficient approximation of the SkL nucleation for realistic sample geometries. An account in the spirit of Bean's critical state model seems necessary [43]. However, unlike superconducting vortices, two scenarios seem plausible for the CP to SkL transition: (i) A SkL front moving toward the center of the sample with nucleation of the skyrmion lines at the edges of the sample and (ii) a nucleation of skyrmion lines at the SkL front using, e.g., the inverse zipper mechanism proposed in Ref. [21].

In conclusion, using $\mathrm{nDI}$, we examined effects of the sample shape on the formation of the SkL phase and distribution in $\mathrm{MnSi}$. We find a macroscopic phase separation of the SkL and CP at the borders of the SkL phase. Our results underline the necessity of considering demagnetization for an unambiguous interpretation of bulk data in terms of intrinsic behavior, in particular for a theoretical modeling of the skyrmion formation. nDI provides a unique tool for future systematic studies of the influence of (i) the sample shape, (ii) the pinning properties, and (iii) the field-temperature history on the SkL nucleation. Moreover, $\mathrm{nDI}$ is also applicable whenever phase separation occurs and the phases can be identified by a Bragg scattering signature. Examples include type II superconductors, polymers, ferroic materials, and samples undergoing structural phase transitions.

We wish to thank S. Mayr, D. Bausenwein, and the FRM II team for technical support and S. Peetermans for fruitful discussions. Funding from the European Union's 7th Framework Programme, NMI3-II Grant No. 283883, DFG TRR80 (Project E1), and ERC Advanced Grant No. 291079 (TOPFIT) is gratefully acknowledged.
[1] F. Jonietz, S. Mühlbauer, C. Pfleiderer, A. Neubauer, W. Münzer, A. Bauer, T. Adams, R. Georgii, P. Böni, R. A. Duine et al., Science 330, 1648 (2010).

[2] T. Schulz, R. Ritz, A. Bauer, M. Halder, M. Wagner, C. Franz, C. Pfleiderer, K. Everschor, M. Garst, and A. Rosch, Nat. Phys. 8, 301 (2012).

[3] N. Romming, C. Hanneken, M. Menzel, J. E. Bickel, B. Wolter, K. von Bergmann, A. Kubetzka, and R. Wiesendanger, Science 341, 636 (2013).

[4] A. Fert, V. Cros, and J. Sampaio, Nat. Nanotechnol. 8, 152 (2013).

[5] X. Zhang, M. Ezawa, and Y. Zhou, Sci. Rep. 5, 9400 (2015).

[6] S. Mühlbauer, B. Binz, F. Jonietz, C. Pfleiderer, A. Rosch, A. Neubauer, R. Georgii, and P. Böni, Science 323, 915 (2009).
[7] W. Münzer, A. Neubauer, T. Adams, S. Mühlbauer, C. Franz, F. Jonietz, R. Georgii, P. Böni, B. Pedersen, M. Schmidt et al., Phys. Rev. B 81, 041203 (2010).

[8] N. Nagaosa and Y. Tokura, Nat. Nanotechnol. 8, 899 (2013).

[9] A. Bauer and C. Pfleiderer, Generic Aspects of Skyrmion Lattices in Chiral Magnets (Springer International Publishing, Cham, 2016), pp. 1-28.

[10] T. Adams, A. Chacon, M. Wagner, A. Bauer, G. Brandl, B. Pedersen, H. Berger, P. Lemmens, and C. Pfleiderer, Phys. Rev. Lett. 108, 237204 (2012).

[11] S. Seki, J.-H. Kim, D. S. Inosov, R. Georgii, B. Keimer, S Ishiwata, and Y. Tokura, Phys. Rev. B 85, 220406 (2012).

[12] T. Kurumaji, T. Nakajima, V. Ukleev, A. Feoktystov, T.-h. Arima, K. Kakurai, and Y. Tokura, Phys. Rev. Lett. 119, 237201 (2017). 
[13] I. Kezsmarki, S. Bordacs, P. Milde, E. Neuber, L. M. Eng, J. S. White, H. M. Ronnow, C. D. Dewhurst, M. Mochizuki, K. Yanai et al., Nat. Mater. 14, 1116 (2015).

[14] Y. Tokunaga, X. Z. Yu, J. S. White, H. M. Ronnow, D. Morikawa, Y. Taguchi, and Y. Tokura, Nat. Commun. 6, 7638 (2015).

[15] A. K. Nayak, V. Kumar, T. Ma, P. Werner, E. Pippel, R. Sahoo, F. Damay, U. K. Rössler, C. Felser, and S. S. P. Parkin, Nature (London) 548, 561 (2017).

[16] X. Z. Yu, Y. Onose, N. Kanazawa, J. H. Park, J. H. han, Y. Matsui, N. Nagaosa, and Y. Tokura, Nature (London) 465, 901 (2010).

[17] X. Z. Yu, N. Kanazawa, Y. Onose, K. Kimoto, W. Z. Zhang, S. Ishiwata, Y. Matsui, and Y. Tokura, Nat. Mater. 10, 106 (2011).

[18] A. Tonomura, X. Yu, K. Yanagisawa, T. Matsuda, Y. Onose, N. Kanazawa, H. S. Park, and Y. Tokura, Nano Lett. 12, 1673 (2012).

[19] S. Heinze, K. von Bergmann, M. Menzel, J. Brede, A. Kubetzka, R. Wiesendanger, G. Bihlmayer, and S. Blugel, Nat. Phys. 7, 713 (2011).

[20] O. Boulle, J. Vogel, H. Yang, S. Pizzini, D. de Souza Chaves, A. Locatelli, T. O. Mentes, A. Sala, L. D. Buda-Prejbeanu, O. Klein et al., Nat. Nanotechnol. 11, 449 (2016).

[21] P. Milde, D. Köhler, J. Seidel, L. M. Eng, A. Bauer, A. Chacon, J. Kindervater, S. Mühlbauer, C. Pfleiderer, S. Buhrandt et al., Science 340, 1076 (2013).

[22] H. Oike, A. Kikkawa, N. Kanazawa, Y. Taguchi, M. Kawasaki, Y. Tokura, and F. Kagawa, Nat. Phys. 12, 62 (2016).

[23] A. Bauer, M. Garst, and C. Pfleiderer, Phys. Rev. Lett. 110, 177207 (2013).

[24] J. Wild, T. N. G. Meier, S. Pöllath, M. Kronseder, A. Bauer, A. Chacon, M. Halder, M. Schowalter, A. Rosenauer, J. Zweck, J. Müller, A. Rosch, C. Pfleiderer, and C. H. Back, Sci. Adv. 3, e1701704 (2017).

[25] F. N. Rybakov, A. B. Borisov, S. Blügel, and N. S. Kiselev, Phys. Rev. Lett. 115, 117201 (2015).

[26] J. C. Loudon, A. O. Leonov, A. N. Bogdanov, M. Ciomaga Hatnean, and G. Balakrishnan, arXiv:1704.06876.

[27] A. Bauer and C. Pfleiderer, Phys. Rev. B 85, 214418 (2012).
[28] I. I. Lobanova, V. V. Glushkov, N. E. Sluchanko, and S. V. Demishev, Sci. Rep. 6, 22101 (2016).

[29] S. V. Grigoriev, N. M. Potapova, E. V. Moskvin, V. A. Dyadkin, C. Dewhurst, and S. V. Maleyev, JETP Lett. 100, 216 (2014).

[30] L. J. Bannenberg, A. J. E. Lefering, K. Kakurai, Y. Onose, Y. Endoh, Y. Tokura, and C. Pappas, Phys. Rev. B 94, 134433 (2016).

[31] H. Wilhelm, M. Baenitz, M. Schmidt, U. K. Rößler, A. A. Leonov, and A. N. Bogdanov, Phys. Rev. Lett. 107, 127203 (2011).

[32] J. Müller, A. Rosch, and M. Garst, New J. Phys. 18, 065006 (2016).

[33] S. Peetermans, A. King, W. Ludwig, P. Reischig, and E. H. Lehmann, Analyst 139, 5765 (2014)

[34] A. S. Tremsin, D. F. R. Mildner, W. B. Feller, and R. G. Downing, IEEE Trans. Nucl. Sci. 51, 1020 (2004).

[35] M. Reiner, A. Bauer, M. Leitner, T. Gigl, W. Anwand, M. Butterling, A. Wagner, P. Kudejova, C. Pfleiderer, and C. Hugenschmidt, Sci. Rep. 6, 29109 (2016).

[36] E. Calzada, F. Grünauer, M. Mühlbauer, B. Schillinger, and M Schulz, Nucl. Instrum. Methods Phys. Res., Sect. A 605, 50 (2009).

[37] A. S. Tremsin, E. H. Lehmann, J. B. McPhate, J. V. Vallerga, O. H. W. Siegmund, B. White, P. White, W. B. Feller, F. C. de Beer, and W. Kockelmann, IEEE Trans. Nucl. Sci. 62, 1288 (2015).

[38] The sickle-shaped pattern originates in the local bending at the edges of the sample in combination with the vertical rocking direction.

[39] R. I. Joseph and E. Schlömann, J. Appl. Phys. 36, 1579 (1965).

[40] T. Adams, S. Mühlbauer, C. Pfleiderer, F. Jonietz, A. Bauer, A. Neubauer, R. Georgii, P. Böni, U. Keiderling, K. Everschor et al., Phys. Rev. Lett. 107, 217206 (2011).

[41] H. Wilhelm, M. Baenitz, M. Schmidt, C. Naylor, R. Lortz, U. K. Rler, A. A. Leonov, and A. N. Bogdanov, J. Phys.: Condens. Matter 24, 294204 (2012).

[42] E. H. Brandt, Phys. Rev. B 58, 6506 (1998).

[43] C. P. Bean, Phys. Rev. Lett. 8, 250 (1962). 